\title{
Entrepreneurial R\&D: An Organizational Design for Enhanced Patent Productivity
}

\author{
Mary Mathew, Urmi Chattopadhyay \\ Department of Management Studies, Indian Institute of Science, Bangalore, India
}

Intellectual property (IP) is showing signs of becoming the principal source of shareholder wealth and competitive advantage and will be the main wealth pusher in days to come. Unlocking IP within an organization requires a focused drive towards result oriented R\&D which is possible with an entrepreneurial orientation within R\&D. The organization design correlates that will maximize patent productivity are the push factors of IP. This paper considers two approaches to understanding the push factors of IP- the understanding an organization has of its patent productivity and diagnosis of the internal processes of an organization that maximize IP.

Patent productivity can be analyzed at two levelsorganizational and patent specific. At the organizational level, the most popular measure is the number of patents generated annually. But this is sometimes inadequate and requires further analysis on an extraction metric in a patent specific way. The commercial potential of patents can be classified into two poles in a linear continuum namely a) no reason patents b) knowledge base developers through to c) crown jewel patents. The other indices are a) Annual Patent Revenue/Annual Patent Expenditure where patent revenue are the revenues from royalty, licensing or sale of patents and expenditure includes all kinds of costs associated with patents b) Patent Budget / R\&D Budget.

The difference in patent output across top twenty organizations according to the number of US patents motivates internal organizational analyses. The internal organizational analyses may contribute to patent pushing and obtaining or patent trying and not getting. In comparison to traditional variables a few patent pushing variables have been identified which are team task roles and empowerment related to IP activities, IP process flow superimposed on $\mathrm{R} \& D$ process $R \& D$ process flow, $H R$ initiatives in recognition and reward of IP, external learning through collaborative $R \& D$ for IP synergy, revenue centric $R \& D$ and extraction metric related assessment of IP. Case Studies done with these variables in the background indicate the serendipitous nature with which a patent emerges. Multinational organizations with R\&D bases in other parts of the globe other than the parent location, handle their IP process in a semi-centralized manner. The current organizational designs fail to empower their teams with IP knowhow like queries on : How to recognize a patent as it comes? When to patent? Where to patent? The case studies throw light on the fact that studying the number of patents only is inadequate and in-depth analysis is required based on these approaches. Redesigning a conscious organization thereafter will enable the organization to get closer to entrepreneurial R\&D which will be by default patent productive. 